\title{
STUDY ON AOTF-BASED NEAR-INFRARED SPECTROSCOPY ANALYSIS SYSTEM OF FARM PRODUCE QUALITY
}

\author{
Xiaochao Zhang ${ }^{*}$, Xiaoan Hu, Yinqiao Zhang, Hui Wang, Hui Zhang \\ ${ }^{1}$ Institute of Mechatronics Technology and Application, Chinese Academy of Agricultural \\ Mechanization Sciences, Beijing, P. R. China100083 \\ * Corresponding author, Address: Box25, No.1 Beishatan, Dewai St, Beijing, 100083, China, \\ Tel: +86-10-64882584,Fax:+86-10-64882652,Email: zxc@caams.org.cn
}

\begin{abstract}
The technology of near-infrared spectroscopy analysis is one of the important ways to test agricultural products inside quality quantitatively. The nearinfrared spectral analysis system which used the technology of sound and light tunable filter (AOTF) can be applied to on-line measurement. The system constructed by AOTF, analysis optimization wavelength together with genetic algorithm, execute data filtering with wavelet analysis technology, process data of signal with second differential and Mahalanobis-distance, establish Partial Least-Squares model, tested the wheat and powdered milk. Reflects the development of the near-infrared spectral analysis system can be applied to the quality of agricultural products preferably.
\end{abstract}

Keywords: Near-infrared, AOTF, Milk Powder, Quality Analyses, Wavelet Analysis, Support Vector Machine

\section{INTRODUCTION}

Information collection, processing and monitoring technology are the key problem of digital agriculture. Near-infrared spectral analysis method is a better way to measure food and milk power quality rapidly (Hao, 2003; Geladi, 1985).

Acousto-optic tunable filter (AOTF) (Pozhar V E et al, 2002) is a new technology appeared in the early 1990s. Using birefringent crystal, by changing the radio frequency to change the wavelength, AOTF made homochromatic ray.

Please use the following format when citing this chapter:

Zhang, X., Hu, X., Zhang, Y., Wang, H. and Zhang, H., 2009, in IFIP International Federation for Information Processing, Volume 295, Computer and Computing Technologies in Agriculture II, Volume 3, eds. D. Li, Z. Chunjiang, (Boston: Springer), pp. 2067-2076. 
The device has no mobile components, with a high scanning speed and better stability (R. TSENKOVA, 2000), so, it is suitable for on-line analysis particularly, is one of the development directions of near-infrared on-line measurement technology (Xu Kexin, 2002). This is a more mature technology in west countries but at the initial stage in china. Since this method can be widely used in quality measurement and control during the process of agricultural production, it will bring new vitality for agricultural equipment industry, and promote the development of quality of agricultural products on-line analysis rapidly with the character of low-cost, non-contact and real-time (Guyon I, 2002).

\section{HARDWARE DESIGN OF AOTF SPECTROMETRY SYSTEM}

\subsection{Structure of the system}

The system is composed of seven departments: rotating sample pool and control unit, high stability light source, AOTF optical system, temperature control unit of detector, detection signal acquisition system, DDS frequency selection control system and computer signal processing system, which is shown in Fig. 1.

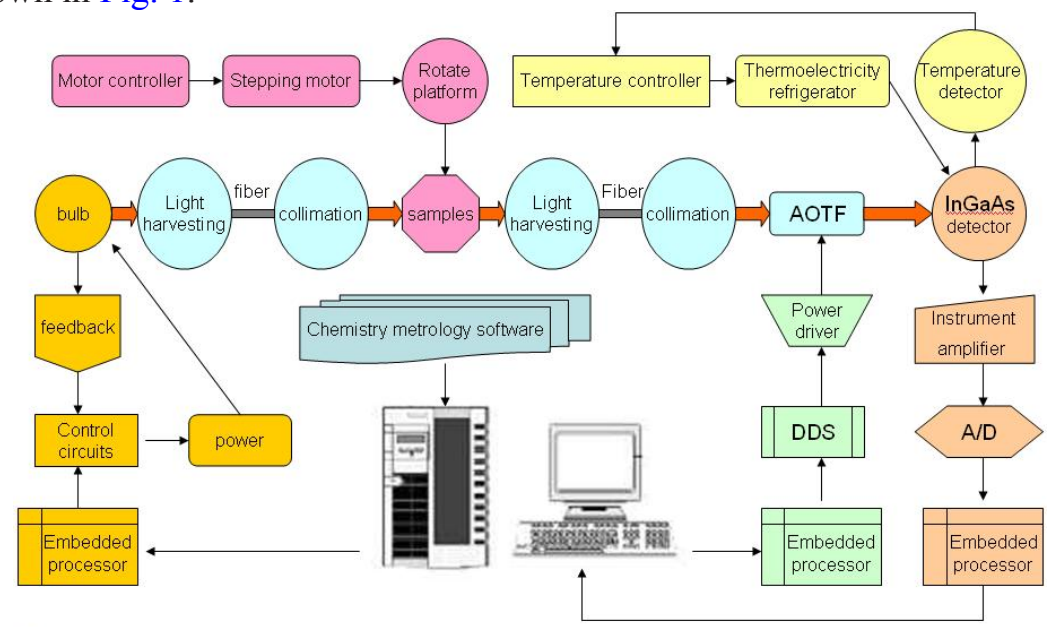

Fig.1: AOTF based spectroscopy analysis system

Use the revolving sample pool to test samples adequately; current-feedbackbased light source control system provide 50 to 150 watts of light; AOTF spectrometry system uses diffuse reflectance measurement method; temperature control unit provide stable environment with an error of \pm 0.2 degree for detectors; the computer issue $20 \mathrm{MHz} \sim 70 \mathrm{MHz}$ inspirit signal through the DDS 
frequency selective circuit; and 16 bit A/D converter collect the corresponding spectrum signals to the computer in real-time.

\subsection{Principle of AOTF}

Sound-light tunable filter is an electro-optical modulator device. Its main principle is to use the Prague dissemination of sound wave to the light insinuated into medium when the sound wave spreading in anisotropic medium. Sound-light tunable filter was composed of single crystal birefringence (common material is $\mathrm{TeO}_{2}$ ), piezoelectric transducers that glued to one side of the single crystal, and high-frequency signal source that used on the piezoelectric transducer. When certain frequency radio signals were imported, AOTF will diffract multicolor light, and choose homochromy light with the wavelength of $\lambda$. The homochromy light wavelength $\lambda$ and radio frequency $f$ has corresponding relations. Choose the second refraction crystal tellurium dioxide designed AOTF, which has the characters as follows:

$\begin{array}{ll}\text { Wavelength: } & 800 \mathrm{~nm} \sim 1700 \mathrm{~nm} ; \\ \text { Aperture: } & \Phi 4 \mathrm{~mm} ; \\ \text { Frequency: } & 20 \sim 70 \mathrm{MHz} ; \\ \text { Spectral resolution: } & 2 \mathrm{~nm} \text { at } 800 \mathrm{~nm} ; \\ 9 \mathrm{~nm} \text { at } 1700 \mathrm{~nm} & \\ \text { Diffraction efficiency: } & 40 \% \text { at } 800 \mathrm{~nm} ; \\ & 20 \% \text { at } 1700 \mathrm{~nm} ; \\ \text { Drive power: } & 1 \mathrm{~W} .\end{array}$

The examination showed that the centre-correlation coefficient between the radio driver frequency and the number of diffraction light waves center.

\subsection{Design of DDS radio frequency driver}

According to the frequency range of $20 \mathrm{MHz} \sim 70 \mathrm{MHz}$ that AOTF RF driver needed, DDS (Direct Digital Synthesizer) clip AD9854 was selected. The chip has a $300 \mathrm{MHz}$ internal clock, 4 to 20 times internal programmable frequency multiplier, which generated an external input clock frequency range of $15 \sim 75$ MHz. In addition, the clip has a $100 \mathrm{MHz}$ parallel interface bus, and an inside orthogonal dual-channel DAC output, working with a variety of programming fashion, and can produce complex signals such as linear and nonlinear FM signals and so on.

The working process is as follows:

(1) Translate digital waveform stored in several forms to Analog waveform by $\mathrm{D} / \mathrm{A}$.

(2) Change the frequency of the output signal by changing the step of Addressing. The step is the incremental phase of digital waveform. The accumulator value is search address; accumulate according to the phase 
accumulator.

(3) Output low-sound analog waveform by low-pass or band-pass filter.

The core department of DDS System is phase accumulator, which is composed of an adder and a phase-based register. Suppose the bit width of the adder is $2^{N}$, the high P-bit accumulator is to search Sin function table, and the frequency of clock is $f_{c}$, then the output frequency is:

$$
f_{\text {out }}=\frac{f_{c}}{2^{N}}
$$

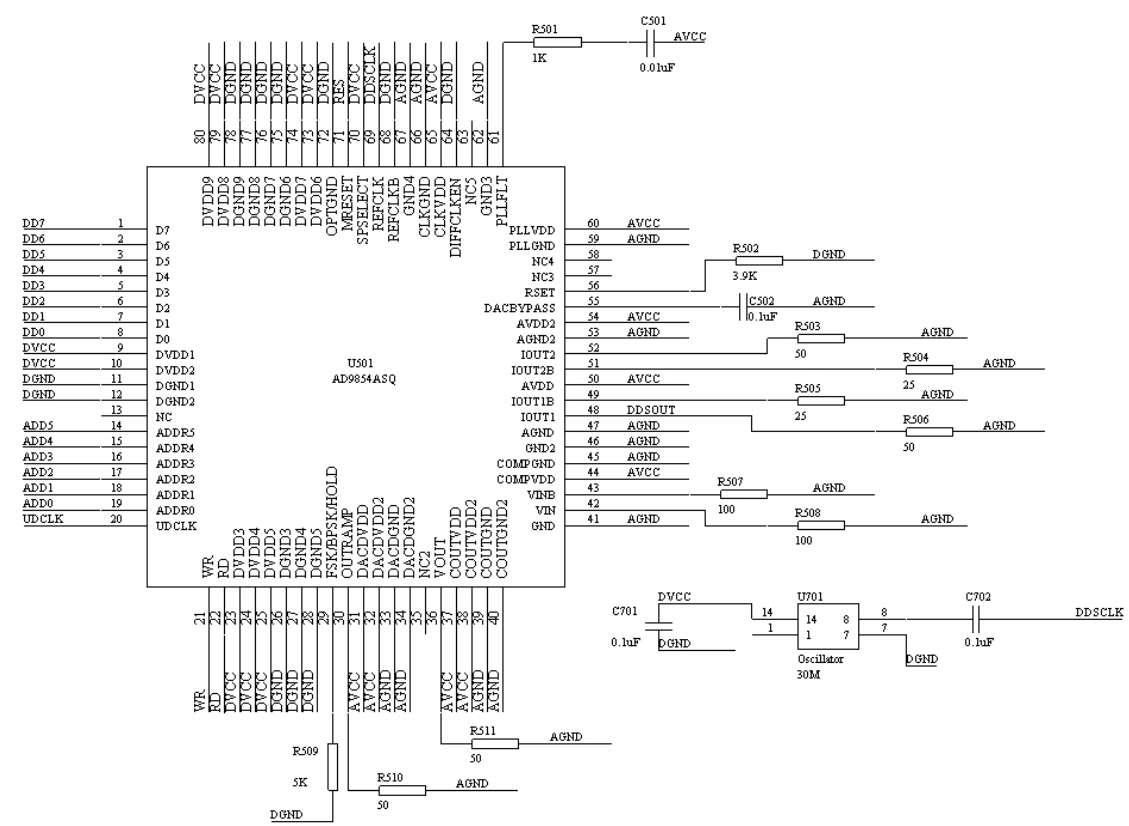

Fig.2: circuits of DDS

\subsection{Design of RF low pass filter}

The requisition of designing an elliptic function low pass filter is: the decay from 0 to $70 \mathrm{MHz}$ should less than $1 \mathrm{~dB}$ and greater than 50 decibels if the frequency bigger than $75 \mathrm{MHz}$, terminal match resistance is $50 \mathrm{ohm}$. The design steps are as follows:

(1) Elliptic function obtained should have an order of seven.

(2) Search for components value from the normalization table according to the value of $A_{P}, A_{S}, \omega_{C}, \omega_{S}$ and $n$.

(3) Calculate components value with integrated calibration formula and design circuit. 


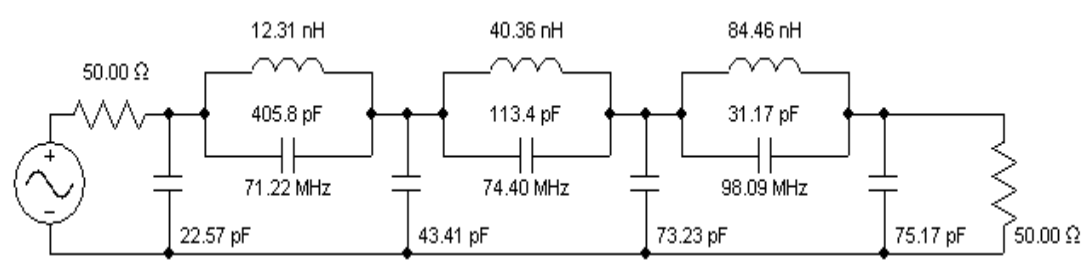

Fig.3: design of filter

The transfer function is as follows:

$$
\frac{5.179 e+077^{*} S^{6}+4.136 e+25 * S^{4}+1.05 e+43^{*} S^{2}+8.607 e+59}{S^{7}+4.013 e+08^{*} S^{6}+5.49 e+17^{*} S^{5}+1.744 e+26^{*} S^{4}+9.871 e+34 * S^{3}+2.315 e+43^{*} S^{2}+5.793 e+51^{*} S+8.607 e+59}
$$

\section{SOFTWARE DESIGN OF AOTF SPECTROMETRY SYSTEM}

To avoid the effect of equipment measuring noise, temperature drifting, light fluctuations and density variety, the following technology were adopt:

(1) Multiplicative scatter correction, standard normal variety, de-trending algorithm and vector normalize.

(2) One-level and two-level window difference technology.

(3) Digital signal processing technology based on wavelet analysis and orthogonal signal correction filter.

(4) Best wavelength automatically search technology based on genetic algorithm.

(5) Principal component based mahalanobis-distance together with spectrum error forecast technology.

(6) Build model whit the technology of support vector machine.

\subsection{Signal processing technology}

Solve the noise interference problems whit the combination of wavelet analysis and orthogonal signal correction. The technology can fully retain effective information when signal filtering processing, and extract objectives related signal. With the characteristics of multi-resolution of wavelet analysis, filter all layers of signal.

Ultimate of orthogonal signal correcting: before establish correction quantitative models; remove the spectrum signal that has nothing to do with concentration matrix with the method of orthogonal mathematical method, and then conduct multiple corrections, so as to simplify the model and improve the ability forecast ability. 
Ultimate of wavelet filter: in wavelet transform, the signal is divided into the cluster overlay of a series wavelet functions, decompose the signal to be analyzed to different scales with the method of multi-scale analysis, and achieve the purpose of signal processing through the hierarchical signal processing and remodeling. Wavelet packet analysis further decomposed not only low frequency but also high frequency part of coefficient; so that it enhanced the time-frequency differentiate.

The formula of fast discrete wavelet packet is:

$$
\left.\begin{array}{l}
d_{j+1,2 n}^{k}=\sum_{j, n}^{l} d_{j, n}^{l} h_{l-2 k} \\
d_{j+1,2 n+1}^{k}=\sum_{l}^{l} d_{j, n}^{l} g_{l-2 k}
\end{array}\right\}
$$

The formula of discrete wavelet packet reconstruction is:

$$
d_{j, n}^{k}=\sum_{l} d_{j+1,2 n}^{l} \bar{h}_{k-2 l}+\sum_{l} d_{j+1,2 n+1}^{l} \bar{g}
$$

With the method of compulsory noise cancellation, set zero to some highfrequency and low frequency coefficient of wavelet packet, and reconstruct the multi-signal.

\subsection{Model construction technology}

Construct model with the method of Support Vector Regression. The use of characteristics of minimum rise, get more stable and precise calibration model.

Suppose the sample set is:

$$
\left(y_{1}, x_{1}\right), \cdots,\left(y_{l}, x_{l}\right), x \in R^{n,} y \in R
$$

And regression function was expressed as the following linear equations:

$$
f(x)=\langle w, x\rangle+b
$$

The best regression function is the minimum value to the following function:

$$
\Phi\left(\mathbf{w}, \xi^{*}, \xi\right)=\frac{1}{2} \mid \mathbf{w} \|^{2}+C\left(\sum_{i=1}^{l} \xi_{i}+\sum_{i=1}^{l} \xi_{i}^{*}\right)
$$

Where $C$ is set penalties values, $\xi$ and $\xi^{*}$ are upper and lower limits of the slack variable.

Use the following insensitive loss function:

$$
L_{\mathrm{e}}(y)=\left\{\begin{array}{cc}
0 & \text { for } \\
|f(\mathbf{x})-y|-\varepsilon & \text { otherwise }
\end{array} \mid f(\mathbf{x})\right.
$$

And the optimize function: 
Study on AOTF-based Near-infrared Spectroscopy Analysis System of 2073 Farm Produce Quality

$$
\max _{\alpha, \alpha^{*}} W\left(\alpha, \alpha^{*}\right)=\max _{\alpha, \alpha^{*}}\left\{\begin{aligned}
- & \frac{1}{2} \sum_{i=1}^{l} \sum_{j=1}^{l}\left(\alpha_{i}-\alpha_{i}^{*}\right)\left(\alpha_{j}-\alpha_{j}^{*}\right) \\
& +\sum_{i=1}^{l} \alpha_{i}\left(y_{i}-\varepsilon\right)-\alpha_{i}^{*}\left(y_{i}+\right.
\end{aligned}\right.
$$

Under the construction of:

$$
\begin{gathered}
0 \leq \alpha_{i} \leq C, \quad i=1, \ldots, l \\
0 \leq \alpha_{i}^{*} \leq C, \quad i=1, \ldots, l \\
\sum_{i=1}^{l}\left(\alpha_{i}-\alpha_{i}^{*}\right)=0
\end{gathered}
$$

Calculate the formula:

$$
\bar{\alpha}^{\alpha}, \bar{\alpha}^{*}=\underset{\alpha, \alpha}{\arg \min _{-*}^{*}}\left\{\begin{array}{l}
\frac{1}{2} \sum_{i=1}^{l} \sum_{j=1}^{l}\left(\alpha_{i}-\alpha_{i}^{*}\right)\left(\alpha_{j}-\alpha_{j}^{*}\right)\left(x_{i}^{T} x_{j},\right. \\
-\sum_{i=1}^{l}\left(\alpha_{i}-\alpha_{i}^{*}\right) y_{i}+\sum_{i=1}^{l}\left(\alpha_{i}+\alpha_{i}^{*}\right) \varepsilon
\end{array}\right.
$$

We get the coefficient $\alpha_{i}$ and $\alpha_{i}^{*}$ of Lagrange equation, and then regression coefficient and constant as follows:

$$
\begin{aligned}
& \bar{w}=\sum_{i=1}^{l}\left(\alpha_{i}-\alpha_{i}^{*}\right) x_{i} \\
& \bar{b}=-\frac{1}{2}\left\langle\bar{w},\left(x_{r}+x_{s}\right)\right\rangle
\end{aligned}
$$

\subsection{Practical technologies for products}

Practical technologies adopted in this thesis include:

Designed Low-power and high-precision portable light source, designed core components of temperature control process; dynamic adjust technology of double-calibration of standard light and background; completed signal and model standardization; Rotating dynamic correction technology and density light automatically tracking measurement method.

\section{APPLICATIONS IN FARM PRODUCE CHARACTER DETECTION}

Examination was done to test the quality of grain and milk powder with developed apparatus. Take dairy products as an example, choose 50 samples 
of milk powder, and set the measure range of wavelength of $800 \sim 1700 \mathrm{~nm}$, points of wavelength is 400 , establish model calibration for pretreatment spectral data of water, ash, acidity and protein respectively. We found that milk ingredients in different wavelength absorption strength were very different, for example, water had a strong absorption peak in the wavelength of $960 \mathrm{~nm}$ and $1440 \mathrm{~nm}$ (Fig. 4).

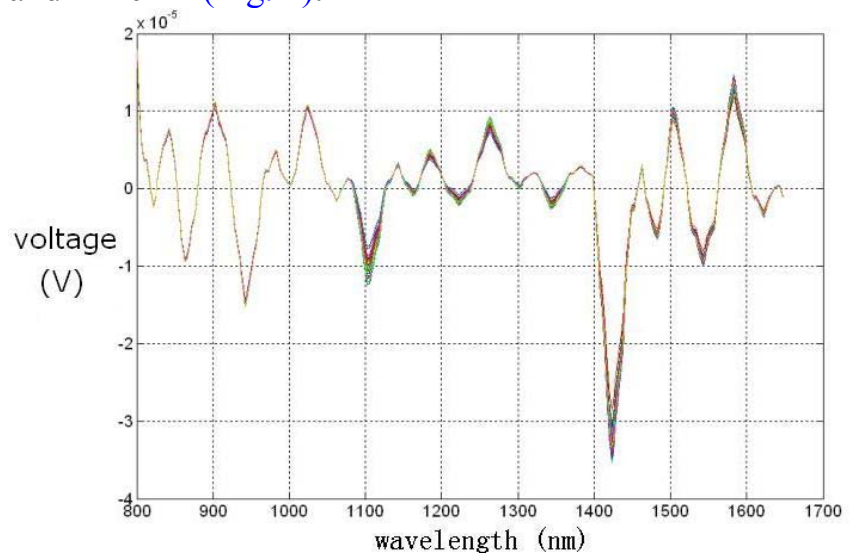

Fig .4: absorption peak of water

The best main components are determined by the square of forecast error of correction aggregate and the predictive residual error sum of squares.

Considering the original baseline drift of the absorption spectrum, a first derivative and second derivative processing technology were adopted. Fig. 5, Fig. 6 and Fig.7 are separately the model correlation of water, ash and protein.

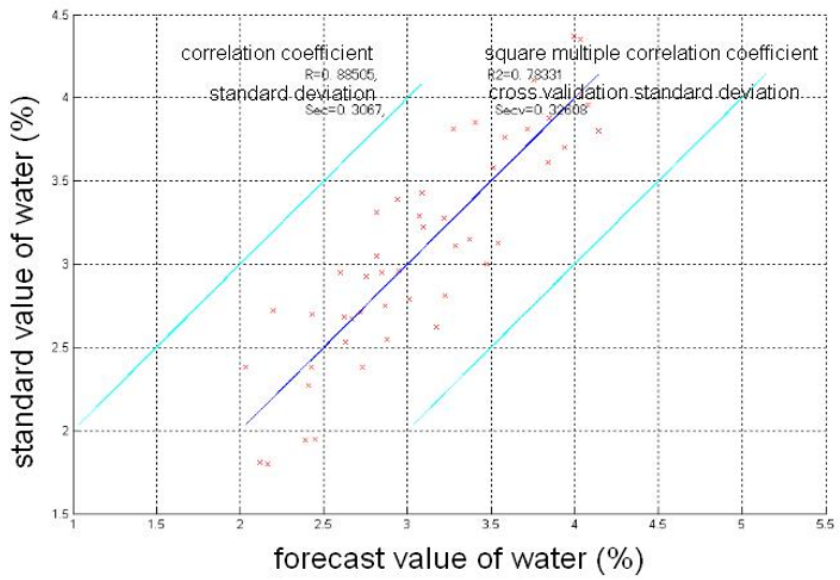

Fig.5: model correlation of water 
Study on AOTF-based Near-infrared Spectroscopy Analysis System of 2075 Farm Produce Quality

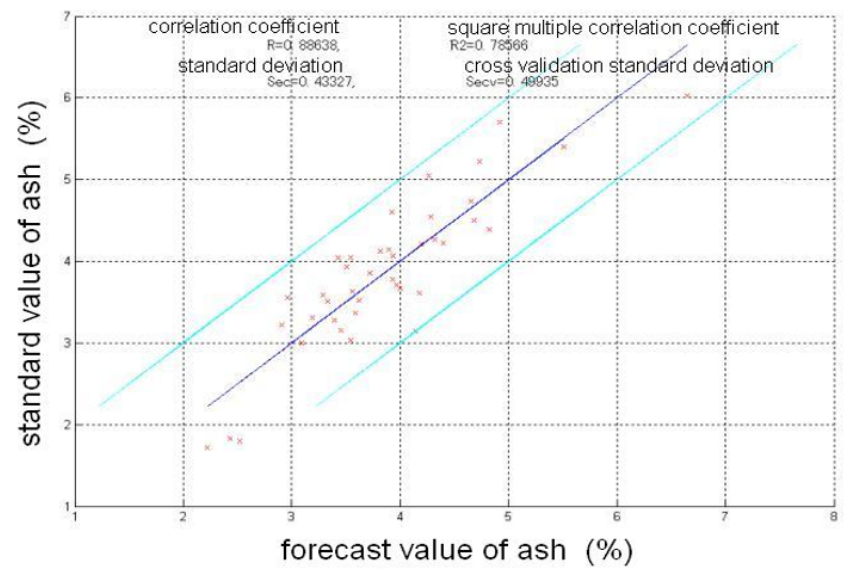

Fig.6: model correlation of ash

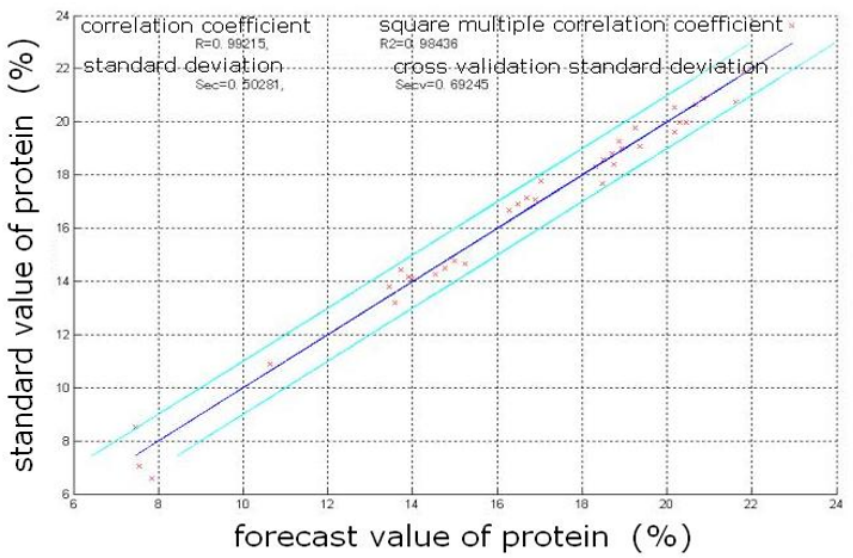

Fig. 7: model correlation of protein

Obligate $20 \%$ samples as forecast ones, four regression models was forecasted separately. Predicted results of protein were shown in table 1 and measurement repeatability test results in table 2 .

Table.1: analysis data of protein

\begin{tabular}{ccccc}
\hline type & Model or not? & number & $\mathrm{R}$ & SEC \\
\hline Revise aggregate & YES & 40 & 0.99215 & 0.5 \\
Forecast aggregate & NO & 10 & 0.9958 & 0.47 \\
\hline
\end{tabular}

Table.2: errors and repetition of each component

\begin{tabular}{ccccc}
\hline Component & Water $(\%)$ & Ash $(\%)$ & Acidity $(\mathrm{T})$ & Protein $(\%)$ \\
\hline Error & 0.4 & 0.6 & 1.2 & 0.7 \\
Repetition & 0.1 & 0.2 & 0.5 & 0.3 \\
\hline
\end{tabular}




\section{CONCLUSIONS}

The examination shown that it was feasible to detect the component of milk powder with the AOTF based principle of near-infrared spectroscopy system. But there were still disparity in errors and repetition with national standard. In order to ensure the accuracy and stability, we will continue to study the following aspects:

(1) Improve the capability of AOTF apartments,

(2) Improve the stability of the frequency signal drive, and,

(3) Enhance the stability and sensitivity of detectors.

The diffuse reflection we used in the spectrum system can detect grain liquid and powder agricultural products directly.

The system is especially suitable for the rapid analysis of large number of repetitive samples, which can detect all the elements in 40 seconds, and the method is simple and repeatable.

\section{REFERENCES}

B Sholkopf ,K Sung, CJ C Burges,et al.Comparing support vector machine with Gaussian kernels to radial basis function classifiers. IEEE Trans. Signal Processing,1997,45:27582765

Guyon I, Weston J, Barnhill S, et al. Gene selection for cancer classfication using support vector machines. Machine Learning,2002, 46(1): 389-422.

Hao Li,Tong Liu,Changyun Wen. All-fiber acousto-optical tunable filter with loop structure. Optical Engineering.2003,42(12):26-31

Johan A.Westerhuis,Sijmen de Jong,Age K.Smilde.Direct orthogonal sighal correction. Chemom.Intell.Lab.Syst.,2001,56:13-25.

O. Svensson,T. Kourti,J. F.MacGregor.An investigation of orthogonal signal correction algorithms and their characterics. J. chemometrics,2002,16:176-188

P Geladi,D MacDougall,H Martens. Linearization and scatter-correction for near-infrared reflectance spectra of meat. Applied Spectrotroscopy,1985,39(03),491-500.

Pozhar V E, Pustovoit V I. Long-pathoptical spectral AOTF-based gas analyzer[J]. SPIE, 2002, 4574:174-178.

R J Barnes, M S Dhanoa,S J Lister.Standard normal variate transformation and de-trending of near-infrared diffuse reflectance spectra. Applied Spectroscopy,1989,43(05):772-777

R. TSENKOVA, Y. OZAKI, et al. Near Infrared Specerosoopy for Biomonhoring: Cow Milk Composition Measurement in a Spectral Region from 1100 to 2400 Namometers[J].J. Anim, Sci., 2000, 78:515 - 521

$\mathrm{Xu}$ kexin, Ni Yong. The optical characteristics of the acousto-optic tunable filter with the equivalent point[J]. Chinese Journal of Lasers, 2002, B11(2):133-138. 